\title{
Electrochemical Detection of Dissolved Sulphur Dioxide Using Bovine Serum Albumin - Theoretical and Experimental Approach
}

\author{
SHRUTHI GIRIDHARAN ${ }^{1}$, JEMINI JOSE² and SREEJA. P. B ${ }^{1 *}$ \\ 1,2Department of Chemistry, CHRIST (Deemed to be University), Hosur Road, Bangalore 560029, India. \\ *Corresponding author E-mail: sreeja.pb@ christuniversity.in
}

http://dx.doi.org/10.13005/ojc/350338

(Received: May 10, 2019; Accepted: June 11, 2019)

\begin{abstract}
In this research work, we discuss the fabrication of an electrochemical sensor that can successfully detect $10^{-3} \mathrm{M}$ concentration of sulphur dioxide. Electrochemical analyses were carried out using glassy carbon electrode modified with the protein - bovine serum albumin. The studies were carried out experimentally and theoretically. The theoretical studies were by molecular docking of bovine serum albumin with sulphur dioxide and experimentally, cyclic voltammetry was performed for different scan rates and concentrations of sulphur dioxide. The results obtained prove that bovine serum albumin has appreciable interaction with sulphur dioxide and hence can be used as a basis for the detection of the same.
\end{abstract}

Keywords: Sulphur dioxide, Electrochemical detection, Bovine serum albumin, Fabrication of electrodes.

\section{INTRODUCTION}

Sulphur dioxide is a compound whose natural state of existence is in the gaseous form. This particular compound is a pollutant that poses a major threat to the environment ${ }^{1}$. Sulphur dioxide gas is a common gas in vehicular emissions. It is also generated in-situ in those food products which have food adulterants comprising sulphite salts, as they on oxidation produce sulphur dioxide. Sulphur dioxide being a polar compound exhibits polarity due to the varying electronegativity in the compound. This property of the compound is employed in development of electrochemical sensor that is in-built with a modified electrode saturated with a compound that has high affinity and exhibits molecular interaction with the sulphur dioxide molecule. The compound that was used to modify the electrode was bovine serum albumin.

The advent of the work on sulphur dioxide began with the study of the effects that sulphur dioxide had on various aspects of plant life processes such as photosynthesis, respiration, and chlorophyll content ${ }^{1}$. The kinetics of the homogeneous gaseous oxidation of sulphur dioxide was studied by Sadek et al., which was performed in the presence of catalysts ${ }^{2}$. A new surface acoustic wave gas sensor was also constructed to detect the presence of sulphur dioxide and this was based on a polyaniline/ $\ln _{2} \mathrm{O}_{3}$ nanofibre composite ${ }^{3}$.

This is an Open Access article licensed under a Creative Commons license: Attribution 4.0 International (CC- BY). Published by Oriental Scientific Publishing Company @ 2018 
The generation of using solid phase electrolyte to detect sulphur dioxide started with the construction of the electrochemical cell that involved selection of a solid electrolyte which can undergo a reversible electrochemical reaction with sulphur dioxide or sulphur trioxide. A novel sensor with sulphate salts of lithium and silver with excellent sensitivity and selectivity was reported by Wolfveis et al., ${ }^{4}$. Sensor that works on the principle of fluorescence was developed to detect sulphur dioxide in 2007, which sense the $\mathrm{SO}_{2}$ by dynamic quenching of benzo ${ }^{5}$. Substituted imidazoline radical was used as a receptor in sulphur dioxide gas sensors. The key principle was on the basis of adsorption. Different molecular structures were simulated and the resultant adsorption complexes obtained with varying functional groups were studied. The compound 2-(4'dimethylyaminophenyl)-4, 4, 5, 5-tetramethylimidazoline-3-oxide-1-oxyl was found to have high sensitivity towards sulphur dioxide. Simulation of the possible adsorption complexes were carried out in DAPTIOO. Conclusion drawn is theoretical calculations based on energies of the adsorption complexes formed can help in selecting a compound with the desired functional group that has sensitivity towards sulphur dioxide ${ }^{6}$. $\mathrm{SnO}_{2}$ thin film $\mathrm{NO}_{2}$ gas sensor was developed and the advantage is that it is a low temperature operating sensor. The tungsten oxide doped tin oxide film was used for the sensing of various oxidizing gases. The selectivity and sensitivity of this technique was high for nitrogen dioxide ${ }^{7}$. Sulphur dioxide detection was expanded to employment of tin dioxide gas sensors. Francis et al., studied that tin oxide undergoes primary surface modification on exposure to sulphur dioxide due to the adsorption of sulphites and sulphates. They proposed two probable mechanisms by which the detection of the gas can be carried out. ${ }^{8}$ Andesite volcanoes were used as a model to monitor the various gases emitted. The primary motive was to detect the quantity of gases emitted and their timely change in composition. Different techniques were employed for the detection of gases like direct sampling, ground based remote sensing like co-relation spectroscopy, OP-FTIR (open-path Fourier transform infrared spectroscopy) and satellite remote sensing of gases. However, comparative study of the techniques prove that COPSEC stands as the simplest method of detection. However, with reference to andesite volcanoes other gases evolution are reliable to confirm the presence of a volcano through the same technique rather than sulphur dioxide $^{9}$. Mcgonigle et al., carried out research on biosensors which discusses about the parameters on constructing a biosensor that could be employed in process control i. e. in the industry of enzyme manufacture etc. For monitoring concentrations, rate of growth etc. The techniques can be extended to development of biosensors for the detection of sulphur dioxide too ${ }^{10}$. Ground-based spectroscopic studies were carried out in volcanic eruptions. The highlights of the research work involve the use of various spectrometric techniques that help in monitoring the composition of gases in hydrothermal vents. Sulphur dioxide is a primary constituent in this and hence the same can be detected in these vents. However, the rate of disappearance of the sulphur dioxide gas in these hydrothermal vents are high and hence cannot be detected easily due to the short exposure time ${ }^{11}$. A novel coumarin based - fluorescent probe for selective detection of bisulphite ions in water and sugar samples was developed. 7-dimethylaminocoumarin-3-aldehyde was synthesised and used for the detection of bisulphite anions in water. ${ }^{12} \mathrm{~A}$ ratiometric fluorescent probe for bisulphite anion by intramolecular charge transfer was developed. The compound used as probe was 4-(1H-Benzimidazol-2 yl) benzaldehyde. Absorption and fluorescence spectroscopy techniques were used to understand the mode of sensing by the probe. The mechanism was by the blocking of the intramolecular charge process as a resultant of which fluorescence will be exhibited. The probe has excellent sensitivity for the bisulphite anion over other anion ${ }^{13}$. Probes were synthesised to study the effect of sulphur dioxide in living cells. The probes synthesized were (E) 2-(3-cyano-4, 5, 5-trimethylfuran-2(5H)-ylidene) malononitrile (TCF) and (E)-2-(3-cyano-4-(2-(7-hydroxy-2-oxo$2 \mathrm{H}$-chromen-3-yl)vinyl)-5,5 dimethylfuran-2(5H)ylide ne)malononitrile. The fluorescent quantum yields were determined by the standard methods available. The compound fluorescein was used as a standard ${ }^{14}$.

A mitochondria-targeted ratiometric fluorescent probe was used to monitor endogenously derived sulphur dioxide derivatives in living cells ${ }^{15}$. Research was also carried out by amperometric detection of sulphur dioxide by means of a carbon nanotube electrode. The electrode was polarized at a voltage of $0.35 \mathrm{~V}$. The analyser that was developed 
can be successfully used for the effective removal of the sulphur dioxide gas from the mixture of $\mathrm{SO}_{2}$ and $\mathrm{NO}_{2}{ }^{16}$. Flash photolysis of sulphur dioxide ${ }^{17}$ and flame atomic absorption spectrometry were employed recently for the same ${ }^{18-20}$.

In this work we report a convenient, simple method for the quantification of dissolved sulphur dioxide using a modified glassy carbon electrode with Bovine serum albumin protein, which is easily available. Theoretically, the extent of interaction of Bovine serum albumin protein with sulphur dioxide was also studied. Nature of the solution opted for the studies was also done based on the theoretical studies. Studies were carried out in different $\mathrm{pH}$ of phosphate buffer solutions and different concentrations of sulphur dioxide.

\section{MATERIALS AND METHODS}

The Bovine serum albumin protein was purchased from Sd fine chemicals. The chemicals hydrochloric acid (L R grade), sodium sulphite and sodium chloride were used without further purification. The electrochemical studies were carried out in an electrochemical workstation (CHI608E). The phosphate buffer solution $(\mathrm{pH}=7.4)$ was prepared using the standard procedure. The reactants for the electrochemical detection were $100 \mathrm{~mm}$ solution of hydrochloric acid $(\mathrm{HCl})$ and $100 \mathrm{~mm}$ solution of sodium sulphite $\left(\mathrm{Na}_{2} \mathrm{SO}_{3}\right)$. The studies were carried out using the bare glassy carbon electrode (GCE) and modified glassy carbon electrode by drop casting bovine serum albumin and egg albumin individually to compare the activity. Cyclic voltammetric studies were carried out for various concentrations of the reaction mixture. Different scan rates were performed in this particular concentration. The molecular docking server used in this particular research work is Patchdock and The open babel software used for the further processing.

\section{RESULTS AND DISCUSSION}

The cyclic voltammogram obtained for determining the electrode activity of the GCE modified with bovine serum albumin has a better current carrying capacity than modified with egg albumin. The current carrying capacity of the modified electrode with bovine serum albumin was compared with the bare glassy carbon electrode.
We could obtain a better carrying capacity with the modified electrode Figure 1.

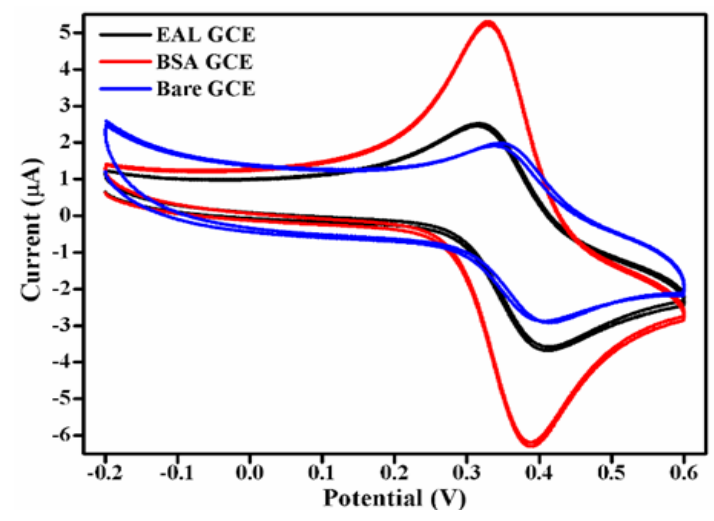

Fig. 1. Cyclic voltammogram for bare glassy carbon electrode (GCE), EAL-GCE and BSA-GCE

The effect of $\mathrm{pH}$ on the electrochemical response was monitored using phosphate buffer solutions of $\mathrm{pH}$ range 3-11. The $\mathrm{pH} 7.4$ was used as gradually with the increase in $\mathrm{pH}$ the electrode surface could possibly get damaged and another problem could be the poor absorption of the analyte due to the high accumulation of the $\mathrm{H}^{+}$and $\mathrm{OH}^{-}$ions on either side of the $\mathrm{pH}$ scale. In high acidic medium, the concentration of $\mathrm{H}^{+}$ions greatly increases and in high alkalinity, the $\mathrm{OH}$ - ions greatly increase. Hence, $\mathrm{pH} 7.4$ was chosen as the right buffer $\mathrm{pH}$ due to its neutral nature and close resemblance to value of physiological $\mathrm{pH}$. The oxidation and reduction peak was clearly obtained for $\mathrm{pH} 7.4$ Figure 2 .

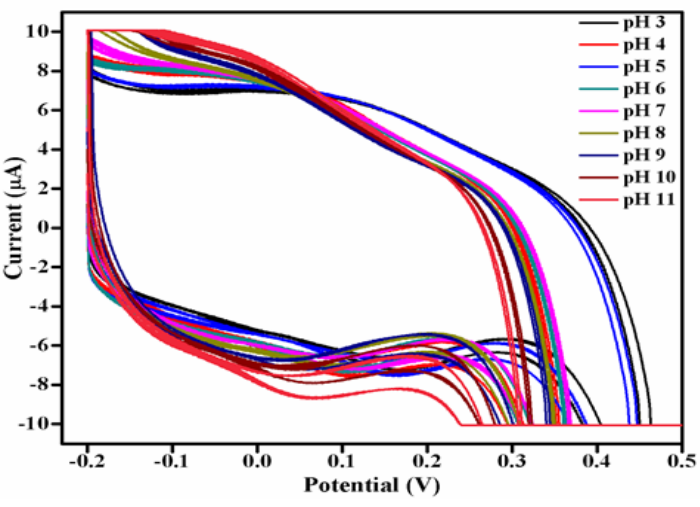

Fig. 2: Cyclic voltammogram obtained for the reaction mixture in different $\mathrm{pH}$

The effect of scan rate was monitored ranging from 10-175 $\mathrm{mVs}^{-1}$ (Fig. 3). The scan rates provide important information on the mechanism of the reaction for the liberation of sulphur dioxide. 
The regression values obtained through the graphs determining the anodic and cathodic current was found to be 0.9244 and 0.967 (Fig. 5a and Fig. 5b), clearly indicating that the co-efficient of correlation is strong. The oxidation peak current at the modified electrode was found to be linearly increasing with the increasing scan rate. Different concentrations of the sulphur dioxide, indicate that with increase in concentration of the analyte the current carrying capacity shows a considerable increase (Fig. 4). The concentration range chosen was $10-100 \mathrm{mM}$. This is a clear indication the specific interaction of sulphur dioxide produced on reaction of hydrochloric acid and sodium sulphite, interacts with the modified electrode surface of bovine serum albumin and hence produces a current with the oxidation and reduction peak. Hence, based on the current produced, the amount of sulphur dioxide can be first standardised and then quantified.

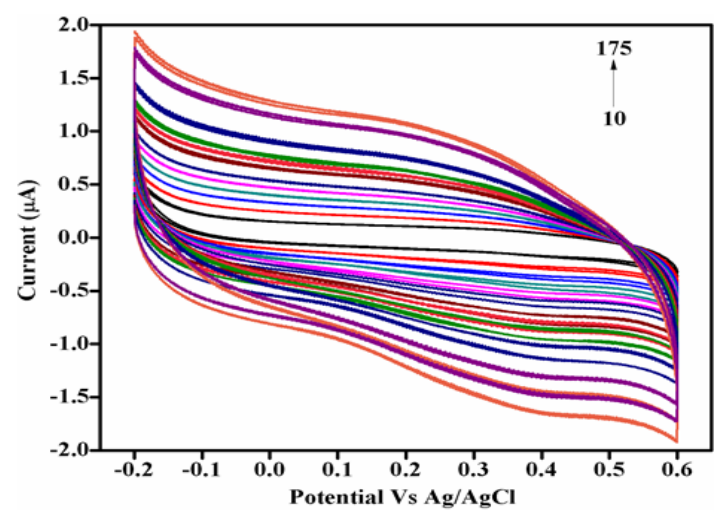

Fig. 3. Graph of Potential Vs Current for different Scan Rates

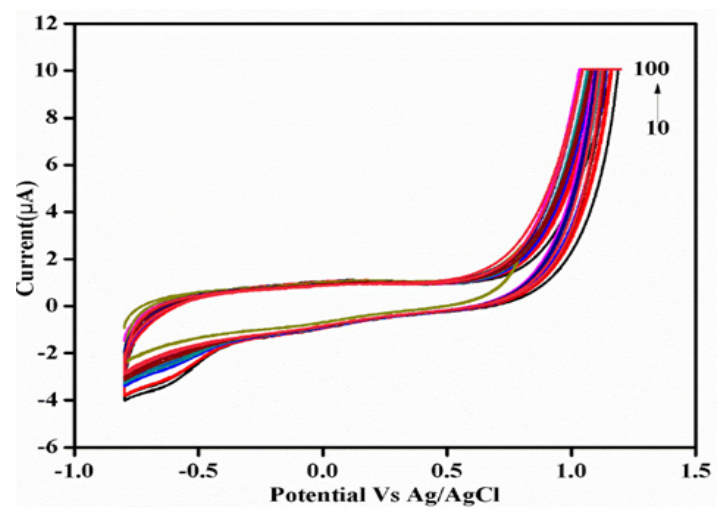

Fig. 4. Effect of concentration of analyte

Theoretical studies were carried out. The molecular docking server used in this particular research work is Patchdock (Fig. 6). The protein molecule that was chosen was bovine serum albumin. The guest molecule is sulphur dioxide. The software used for this particular file conversion was Open Babel. The docking score obtained was considerably good for further studies. This is considered as evidence for interaction between sulphur dioxide and bovine serum albumin.

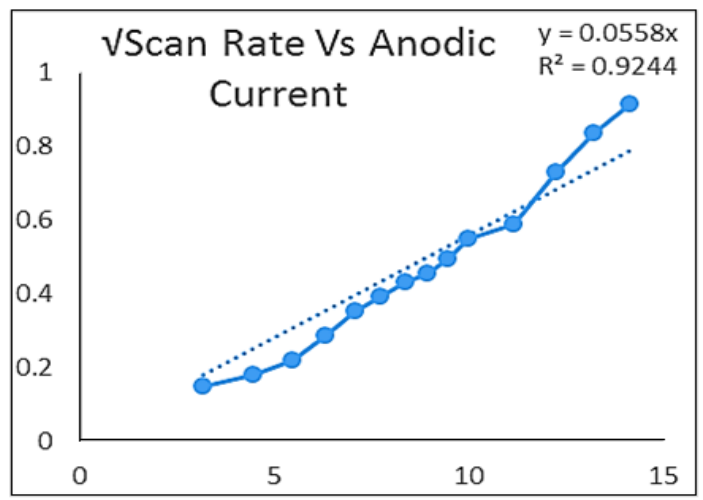

Fig. 5. (a) Linear Graph of Square root of Scan rate Vs Anodic Current

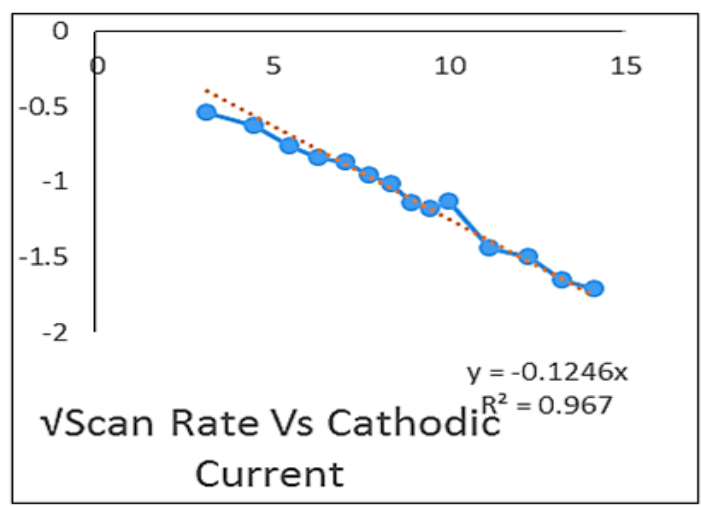

Fig. 5. (b) Linear Graph of Square root of Scan rate Vs Cathodic Current

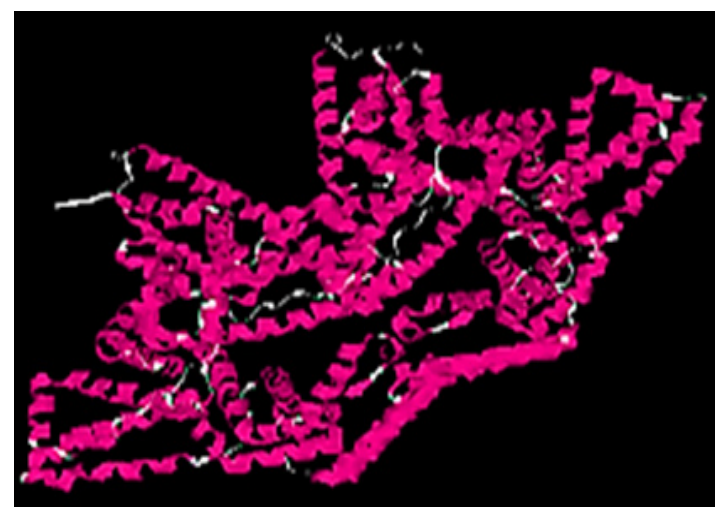

Fig. 6. Molecular Docking structure of BSA and Sulphur Dioxide 


\section{CONCLUSION}

The studies carried out support the hypothesis for the interaction of bovine serum albumin with sulphur dioxide, hence, it can be concluded that the glassy carbon electrode modified with bovine serum albumin can serve as a potential electro-active surface for the detection of low concentration pollutant like sulphur dioxide in the ppm concentration. The bovine serum albumin can be used for the quantification of sulphur dioxide electrochemically. The results obtained by the theoretical studied support that there is an appreciable interaction between bovine serum albumin and sulphur dioxide and hence the two can be used for the electrochemical studies. Experimentally, the characteristic interaction of the bovine serum albumin with the sulphur dioxide was clearly studied due to the variation in the current produced. This variation was studied under the area of effect of scan rates and in the effect of concentration of the analyte. The increasing current with the increasing concentration proved that there is a positive interaction between the host and guest molecule. The plot of the square root of scan rate Vs the cathodic and anodic current respectively is a supportive proof that bovine serum albumin can be used for the quantification of sulphur dioxide electrochemically.

The future of this research work can be looked upon as a work that could possibly pave to future improvements in the field of electrochemical detection and a cheap, reliable and easy to reproduce sensor for sulphur dioxide.

\section{ACKNOWLEDGEMENT}

The authors acknowledge the support rendered by Dr James Arulraj and Dr Manoj B, CHRIST (Deemed to be University).

\section{Confilicts of Interest}

The authors declare no confilict of interest.

\section{REFERENCES}

1. Brookes, C. A.; O' Neill, J. B. Redfern,; B. A.W.; Proc. R. Soc. Lond. A., 2016, 322, 73-88.

2. Sadek, A.Z.; Wlodarski, W.; Shin, K.; Kaner, R.B.; Kalantar-Zadeh, K.; Nanotechnology., 2006, 17, 4488-4492.

3. Worrell, W. L; Liu, Q. G.; J. Electroanal. Chem., 1984, 168, 355-362.

4. Wolfbeis, O. S.; Sharma,A.; Anal. Chim. Acta., 1988, 208, 53-58.

5. Dultsev, F. N.; Sveshnikova,L.L; Sensors Actuators, B Chem., 2007, 120, 434-438.

6. Kaur, J.; Roy, S. C.; Bhatnagar, M. C.; Sensors Actuators, B Chem., 2007, 123, 1090-1095.

7. Girardin,, D.; Berger, F.; Chambaudet, A.; Planade, R.; Sensors Actuators, B Chem., 1997, 43, 147-153.

8. A. Volcanoes, Francis, B. P.; Horrocks,L.; Philos. Trans. R. Soc.Lon., 2016, 358, 1567-1584.

9. Wilson, C. J. N.; Philos. Trans. R. Soc.Lon., 2018, 314, 229- 310.

10. Mcgonigle, A. J. S., Philos. Trans. R. Soc. A Math. Phys. Eng. Sci., 2005, 363, 2915-2929.
11. Yang, Y., Sensors Actuators, B Chem., 2012, 166, 665-670.

12. Sun, Y.;Zhao,D.; Fan,S.; Duan,L.; Li,R.; J. Agric. Food Chem., 2014, 62, 3405-3409.

13. Wu,M.Y.; Li,K.; Li,C.Y.; Hou,J.T.;Yu,X.Q.; Chem. Commun., 2014, 50, 183-185.

14. Xu,W.; Teoh, C. L; Peng,J.; Su,D.; Yuan,L.; Chang,Y.T.; Biomaterials., 2015, 56, 1-9.

15. O'Brien, J.A.; Hinkley,J.T.;Donne,S.W.; J. Electrochem. Soc., 2012., 159, F585-F593.

16. Cheng,X.; Jia, H.; Feng,J.; Qin,J.; Li,Z.; Sensors Actuators, B Chem., 2013, 184, 274-280.

17. Zare-Dorabei,R.; Boroun,S.; Noroozifar, M.; Talanta., 2018, 178, 722-727.

18. Çiftyürek,E.; Sabolsky,K.; Sabolsky,E.M.; Sensors Actuators, B Chem., 2016, 237, 262-274.

19. Eranna,G.; Joshi, B. C.; Runthala,;Gupta, D. P, Crit. Rev. Solid State Mater. Sci., 2004, 29 , 111-188.

20. Jemini, J.; Riya, D.; Sreeja, P.B.; Orient. J. Chem., 2017, 33, 1438-1446. 